\title{
Uso de fertilizantes y compuestos orgánicos en dos variedades de arroz secano (Oryza sativa)
}

Use of fertilizers and organic compounds in two varieties of dry land rice

(Oryza sativa)

\author{
Castro Castañeda Martin Alejandro ${ }^{1}$, Loaiza Velásquez Luis Emmanuel ${ }^{1} \mathrm{y}$ \\ Bastidas López Harold² \\ ${ }^{1}$ Ing. Agrónomos, Universidad de los Llanos y \\ ${ }^{2}$ Ing. Agrónomo, MSc., Docente Universidad de los Llanos \\ hbastidas@unillanos.edu.co
}

Recibido 25 de Enero 2017, Aceptado 26 de Mayo 2017

\section{RESUMEN}

Las enfermedades en el arroz (Oryza sativa) son una de las principales limitantes de su productividad causando inestabilidad en el rendimiento de este cereal, por lo tanto, se recomienda un buen sistema de fertilización. El objetivo del presente estudio fue evaluar dos variedades de arroz: Fedearroz 68 (VF 68) y Victoria 10-39 (VV10-39) con seis diferentes mezclas de fertilizantes orgánicos y químicos, realizado en Villavicencio (Meta); las variedades se sembraron en un área de 1080 $\mathrm{m}^{2}$, con semilla certificada, haciendo el control de malezas, plagas y enfermedades de acuerdo al grado de infestación que se presentó. La fertilización se inició 15 días después de la siembra, posteriormente se fertilizó cada 15 días hasta completar un total de tres o cuatro aplicaciones. Los tratamientos $(\mathrm{T})$ fueron: $1=45 \%$ orgánico con carbono; $2=90 \%$ orgánico sin carbono; $3=100 \%$ químico con urea (tradicional); $4=100 \%$ químico sin urea (otra alternativa tradicional); $5=$ $45 \%$ orgánico con DAP $\left(\mathrm{P}_{2} \mathrm{O}_{5}\right)$ y $6=90 \%$ orgánico sin DAP. FO1= fertilizante orgánico 1 ( $\mathrm{N}$ orgánico); FO2= fertilizante orgánico 2 ( $\mathrm{N}$ ureico); FQ1= fertilizante químico 1 (boro); FQ2= fertilizante químico 2 (urea); FQ3= fertilizante químico 3 (DAP); FQ4= fertilizante químico 4 (carbono); FQ5= fertilizante químico 5 (cloruro de potasio) y $\mathrm{FQ} 6=$ fertilizante químico 6 triple $15\left(15 \mathrm{~N} 15 \mathrm{~K}_{2} \mathrm{O}\right.$ y $\left.15 \mathrm{P}_{2} \mathrm{O}_{5}\right)$. Para la recolección de las muestras se construyeron marcos de $2 \times 3\left(6 \mathrm{~m}^{2}\right)$ para ubicar 
en todos los bloques del ensayo las dos variedades; se cortaron las panículas para así determinar el peso del grano, además se recogieron 10 panículas por bloque para evaluar el peso del grano lleno y vaneado, y con ello estimar porcentaje de vaneamiento y manchado en grano, también se estimó el porcentaje de incidencia de las siguientes patógenos: Sarocladium oryzae, Helminthosporium, Ácaros, Gaenomices, Pyricularia oryzae y virus hoja blanca (VHB). Los componentes de rendimiento que se analizaron suministraron datos que permiten concluir que la fertilización realizada con el T4 en VV 10-68 es la que mejor aporte nutricional dio a la planta, generando un óptimo desarrollo del cultivo, permitiéndole ser el tratamiento con mayor tolerancia a los problemas fitosanitarios que se presentaron, mientras que en $\mathrm{T} 1$ la fertilización no fue suficiente para generar una buena nutrición y así afrontar los problemas fitosanitarios, además su desarrollo fisiológico fue retrasado y débil. En VF 68 el T5 mostró los mejores resultados en todas las variables evaluadas, en tanto que T6 fue el de menor rendimiento y fue susceptible a la mayoría de problemas fitosanitarios.

Palabras clave: Cereal, manejo, biofertilizante, calidad, rendimiento.

\section{ABSTRACT}

The diseases in rice (Oryza sativa) are one of the main constraints of their productivity causing instability in the yield of this cereal, therefore a good fertilization system is recommended. The objective of the present study was to evaluate two rice varieties: Fedearroz 68 (VF 68) and Victoria 10-39 (VV10-39) performed in Villavicencio (Meta); the varieties were planted an area of $1080 \mathrm{~m}^{2}$, with certified seed, making weed control, pests and diseases according to the degree of infestation that was presented. The fertilization began 15 days after sowing, subsequently was fertilized every 15 days Up to complete a total of three or four applications. The treatments $(T)$ were: $1=45 \%$ organic with carbon; $2=90 \%$ organic without carbon; $3=100 \%$ chemical with urea (traditional); $4=100 \%$ chemical without urea (another traditional alternative); $5=45 \%$ organic with DAP $\left(\mathrm{P}_{2} \mathrm{O}_{5}\right)$ and $6=90 \%$ organic without DAP. FO1= organic fertilizer 1 (organic $\mathrm{N}$ ); $\mathrm{FO} 2=$ organic fertilizer 2 ( $\mathrm{N}$ ureic); FQ1= chemical fertilizer 1 (boron); FQ2= 
chemical fertilizer 2 (urea); FQ3 = chemical fertilizer 3 (DAP); FQ4= chemical fertilizer 4 (carbon); FQ5= chemical fertilizer 5 (potassium chloride); and FQ6 = chemical fertilizer 6 triple $15\left(15 \mathrm{~N} 15 \mathrm{~K}_{2} \mathrm{O}\right.$ and $\left.15 \mathrm{P}_{2} \mathrm{O}_{5}\right)$. For the collection of samples frames of $2 \times 3\left(6 \mathrm{~m}^{2}\right)$ were built to locate the two varieties in all test blocks; the panicles were cut to determine grain weight, in addition, 10 panicles were collected per block to evaluate full and weighted grain weight, to evaluate the weight of the full and banned grain, and with it estimate percentage of banishing and stained in grain, also the incidence rate of the following pathogens was estimated: Sarocladium oryzae, Helminthosporium, Ácaros, Gaenomices, Pyricularia oryzae and white-leaf virus (WLV). The performance components that were analyzed provided data that allow the conclusion that fertilization with T4 in VV 10-68 is best nutritional contribution gave the plant, generating an optimal development of the crop, allowing it to be the treatment with greater tolerance to the phytosanitary problems that arose, while in $\mathrm{T} 1$ the fertilization was not enough to generate a good nutrition and thus meet the phytosanitary problems, in addition its physiological development was delayed and weak. In VF 68 the T5 showed the best results in all variables evaluated, while T6 was the lowest performing and was susceptible to most phytosanitary problems.

Keywords: Cereal, management, bio-fertilizer, quality, performance.

\section{RESUMO}

As doenças do arroz (Oryza sativa) são uma das principais limitantes de seu produtividade causando instabilidade no desempenho deste cereal, portanto, um bom sistema de fertilização é recomendado. O objetivo deste estudo foi avaliar duas variedades de arroz: Fedearroz 68 (VF 68) e Victoria 10-39 (VV10-39) com seis diferentes misturas de fertilizantes orgânicos e químicos, realizado em Villavicencio (Meta); as variedades foram plantadas em uma área de $1.080 \mathrm{~m}^{2}$, com sementes certificadas, tornando o controle de ervas daninhas, pragas e doenças de acordo com o grau de infestação que foi apresentada. A fertilização foi iniciada 15 dias após o plantio, posteriormente, foi fertilizado cada 15 dias, até completar um total de três ou quatro aplicações. Os tratamentos $(T)$ foram: $1=45 \%$ 
orgánico com carbono; 2=90\% orgánico sem carbono; 3=100\% químico com ureia (tradicional); $4=100 \%$ químico sem ureia (outra alternativa tradicional); $5=$ $45 \%$ orgánica com DAP $\left(\mathrm{P}_{2} \mathrm{O}_{5}\right)$ e $6=90 \%$ orgânico sem DAP. FO1= fertilizante orgânico 1 ( $\mathrm{N}$ orgânico); FO2= fertilizante orgânico 2 ( $\mathrm{N}$ ureico); FQ1= fertilizante químico 1 (boro); FQ2 = fertilizante químico 2 (ureia); FQ3= fertilizante químico 3 (DAP); FQ4= fertilizante químico 4 (carbono); FQ5= fertilizantes químicos 5 (cloreto de potássio); e FQ6= fertilizante químico 6 triplo 15: 15 ( $15 \mathrm{~K}_{2} \mathrm{O}$ e 15 $\left.\mathrm{P}_{2} \mathrm{O}_{5}\right)$. Para recolher amostras foram construídos quadros de $2 \times 3\left(6 \mathrm{~m}^{2}\right)$ para localizar em todos os blocos de teste as duas variedades; as panículas foram cortadas para determinar o peso dos grãos, também 10 panículas foram recolhidas por bloco para avaliar o peso do grão cheio e vaneado, e, assim, estimar percentagem de vaneamiento e manchado em grão, também foi estimado a percentagem de incidência dos seguintes patogénios: Sarocladium oryzae, Helminthosporium, Ácaros, Gaenomices, Pyricularia oryzae e Vírus folha branco (VFB). Os componentes de rendimento que foram analisados forneceram dados que permitem concluir que a fertilização realizada com T4 VV 10-68 é a que melhor contribuição nutricional deu a planta, gerando um desenvolvimento óptimo o cultivo permitindo ser o tratamento com uma maior tolerancia aos problemas fitossanitários que foram apresentados, enquanto que em T1 a fertilização não foi suficiente para gerar uma boa nutrição e, assim, atender aos problemas fitossanitários, além seu desenvolvimento fisiológico foi atrasado e fraco. Em VF 68 o T5 apresentou os melhores resultados em todas as variáveis avaliadas, enquanto T6 foi o de menor desempenho e foi suscetível a maioria dos problemas fitossanitários.

Palavras-chave: Cereais, gestão, bio-fertilizantes, qualidade, desempenho.

\section{INTRODUCCIÓN}

El arroz (Oryza sativa), es nativo del sureste asiático, se han hallado pruebas de su cultivo desde antes del año 6000 A.C. en una caverna en Tailandia y antes del año 5000 A.C. en China, donde se desarrolló su cultivo desde las tierras bajas hasta las más altas; probablemente hubo varias rutas por las cuales, desde Asia 
introdujeron el cultivo del arroz a otras partes del mundo (Chica et al., 2016). Se han generado en los últimos años, nuevas variedades mediante una gradual renovación de las más antiguas, rescatando las mejores características, pero a la vez se ha ocasionado la desaparición de determinadas variedades, pues las nuevas ofrecen mejores rendimientos y calidad de grano, además de una mayor producción y resistencia a enfermedades, esto a través de los programas de mejoramiento genético basados en la producción de plantas de arroz dihaploides, mediante el cultivo de anteras de plantas obtenidas a partir de cruzamientos previos (Rippstein et al., 2001).

Colombia cuenta con dos sistemas de producción: mecanizado que representa el $94 \%$ de la zona de los arrozales y tradicional (manual) con el que se produce el $6 \%$ del arroz en el país; el sistema mecanizado se subdivide en arroz de riego y de secano, el primero su agua proviene de distritos de riego por bombeo, mientras que en el segundo, su agua son obtenidas de las lluvias recuperadas a través de canales de drenaje, más del $70 \%$ de la producción corresponde al arroz de riego (Castilla et al., 2017).

Las enfermedades son una de las principales limitantes de la productividad del arroz y una causa de la inestabilidad del rendimiento de este cereal, también es importante resaltar que las inadecuadas prácticas de fertilización, preparación del suelo y uso del agua, lo mismo que el uso de variedades no adaptadas a las condiciones agroclimáticas y deterioro genético, conllevan a un bajo rendimiento de este cultivo. Como en todos los países productores de arroz, Colombia no es la excepción y cuenta con bajos rendimientos en la producción, causado por las limitaciones mencionadas anteriormente; por este motivo diferentes programas de investigación en Colombia están produciendo nuevos materiales como alternativa a cada una de las limitantes que más afectan el rendimiento, para posteriormente convertirlas en variedades comerciales que sean asequibles a los productores (Duwayri y Van Tran, 1999).

Variedades de arroz como Fedearroz 68 y Victoria son una alternativa viable en la lucha contra las principales causas del bajo rendimiento, puesto que estas se 
adaptan fácilmente condiciones climatológicas de Colombia y son resistentes a patógenos como Pyricularia grisae y virus de la hoja blanca, además son tolerantes a insectos fitófagos, pero no muestran susceptibilidad a herbicidas, lo cual afecta en gran medida el rendimiento del cultivo de arroz; claro está que para que se llegue a dar una buena producción es muy importante llevar a cabo un buen plan de fertilización, ya sea con productos químicos u orgánicos (FEDEARROZ, 2011).

En este sentido surgen preguntas como: qué tipo de fertilizante usar, o cual es el más efectivo, o si es mejor un fertilizante orgánico o químico compuesto; por este motivo este trabajo tiene como objetivo realizar evaluaciones de diferentes dosis de estos fertilizantes en dos variedades de arroz secano (Oryza sativa) Victoria 10-39 y Fedearroz 68, para que sirva de guía a los productores, que piensen sembrar alguna de dichas variedades en la región de la Orinoquia Colombiana.

Aunque existe una guía básica para fertilizar el arroz en esta región, es necesario ajustarla a las condiciones de cada sitio de acuerdo al resultado del análisis de suelos, de esta forma, la variedad expresará su potencial como respuesta a la oportuna fertilización siguiendo las recomendaciones del sistema de fertilización arrocera (SIFA) (Tinoco y Acuña, 2009).

Debido a la precocidad de estas variedades Victoria 10-39 y Fedearroz 68, se recomienda manejar la nutrición de acuerdo a las etapas de desarrollo, por lo cual el empleo de abonos, se debe hacer en las primeras etapas de crecimiento, y de esta forma la variedad expresará su potencial como respuesta a la oportuna fertilización, es así que el $80 \%$ del nitrógeno debe fraccionarse entre el preabonamiento y antes de inicio del primordio floral (IPF) (FEDEARROZ, 2011); en el segundo semestre del año, la dosis de nitrógeno puede incrementarse.

La aplicación de fosforo se recomienda preferiblemente en pre-siembra incorporada especialmente en el riego, se puede distribuir $50 \%$ en pre-siembra y $50 \%$ al inicio de macollamiento (IM). El Potasio (K), se fracciona en las tres 0 cuatro abonadas con el nitrógeno desde la pre-siembra. Los micronutrientes, el 
calcio, magnesio y el azufre se aplican de acuerdo al análisis químico del suelo. Los elementos menores incorporarlos al momento de la siembra, y los secundarios fraccionados en la fase vegetativa; no olvidar que el plan de fertilización debe formularse con base en el análisis de suelos, la oferta ambiental y el criterio del asistente técnico (FEDEARROZ, 2015).

Las variedades Victoria 10-39 y Fedearroz 68 se generaron para la región de la Orinoquia Colombiana, y se caracterizan por su precocidad y alto rendimiento, así como la tolerancia al virus de la hoja blanca y a Pyricularia grisae, los más temibles patógenos de los campos arroceros, son resultado de dos líneas que hicieron parte de una serie de 1000 materiales de arroz entregados por el "Fondo Latino-americano para Arroz de Riego" en el año 1997; estas variedades fueron evaluadas y aprobadas por el Instituto Colombiano Agropecuario, ICA y Federación Nacional de Arroceros (FEDEARROZ), fueron comercializadas y sembradas en esta región Colombiana; tienen un buen comportamiento tanto en riego como en secano, presentan buena calidad industrial y culinaria, y tienen un periodo de aproximado 111 días de germinación hasta la cosecha. La variedad Victoria 68 es moderadamente resistente a Pyricularia Oryzae tanto en cuello como hoja, también igual comportamiento con patógenos como Sarocladium Oryzae y Rhizoctonia solani (FEDEARROZ, 2015)

\section{METODOLOGÍA}

\section{Ubicación}

El presente estudio se realizó en la granja de la Universidad de los Llanos, localizada en la vereda Barcelona a $14 \mathrm{~km}$ del municipio de Villavicencio, departamento del Meta, con una temperatura promedio anual de $25,7^{\circ} \mathrm{C}$ y las precipitaciones suelen estar alrededor de los $4.008 \mathrm{~mm}$ y humedad relativa promedio anual de $76 \%$ (IDEAM, 2016). 


\section{Manejo de los cultivos}

La siembra se realizó en un área de $600 \mathrm{~m}^{2}$ para la variedad Victoria 10-39 y 480 $\mathrm{m}^{2}$ para Fedearroz 68. Las cantidades de fertilizantes orgánico y químico compuesto que se utilizaron se especifican en las Tablas 1 y 2 . La siembra se realizó al voleo con una densidad de $200 \mathrm{~kg}$ por hectárea de semilla certificada de cada una de las variedades que se trabajaron; se inició la fertilización 15 días después de la siembra, posteriormente se fertilizo cada 15 días hasta completar un total de tres o cuatro aplicaciones (Tabla 3). El control de malezas, plagas y enfermedades se hizo de acuerdo al grado de infestación que se presentó.

Tabla 1. Fertilizantes orgánicos (FO) utilizados. composición en porcentaje

\begin{tabular}{ccc}
\hline Composición & $\begin{array}{c}\text { Fertilizante con } \\
\text { N orgánico (FO1) }\end{array}$ & $\begin{array}{c}\text { Fertilizante con N } \\
\text { ureico (FO2) }\end{array}$ \\
\hline Nitrógeno total $(\mathrm{N})$ & 9 & 5 \\
Nitrógeno amoniacal $(\mathrm{N})$ & 8.5 & 3 \\
Nitrógeno orgánico $(\mathrm{N})$ & 0.5 & - \\
Nitrógeno ureico & - & 2 \\
Fósforo total $\left(\mathrm{P}_{2} \mathrm{O}_{5}\right)$ & 20 & 4 \\
Potasio total $\left(\mathrm{K}_{2} \mathrm{O}\right)$ & 6 & 23 \\
Calcio total $(\mathrm{CaO})$ & 7 & 6 \\
Magnesio total $(\mathrm{MgO})$ & 3 & 4 \\
Silicio total $(\mathrm{SiO})$ & 8 & 7 \\
Carbono orgánico oxidable total & 6 & 5 \\
Zinc $(\mathrm{Zn})$ & 1 & - \\
Humedad & 14.8 & 10.25 \\
pH en $10 \%$ & 7.95 & 8.67 \\
Densidad a $20^{\circ} \mathrm{C}(\mathrm{g} / \mathrm{cc})$ & 0.78 & 0.86 \\
Conductividad eléctrica $(1: 100) \mathrm{ds} / \mathrm{m}$ & 6.26 & 7.15 \\
Capacidad de intercambio catiónico $(\mathrm{meq} / 100 \mathrm{~g})$ & 21.80 & 14.0 \\
\hline
\end{tabular}

Los tratamientos $(\mathrm{T})$ fueron: $1=45 \%$ orgánico con carbono; $2=90 \%$ orgánico sin carbono; $3=100 \%$ químico con urea (tradicional); $4=100 \%$ químico sin urea (otra alternativa tradicional); $5=45 \%$ orgánico con DAP $\left(\mathrm{P}_{2} \mathrm{O}_{5}\right)$ y $6=90 \%$ orgánico sin DAP. FO1= fertilizante orgánico 1 ( $\mathrm{N}$ orgánico); FO2 fertilizante orgánico 2 ( $\mathrm{N}$ ureico); FQ1= fertilizante químico 1 (boro); FQ2= fertilizante químico 2 (urea); 
FQ3 = fertilizante químico 3 (DAP); FQ4= fertilizante químico 4 (carbono); FQ5= fertilizante químico 5 (cloruro de potasio) y FQ6= fertilizante químico 6 triple 15 $\left(15 \mathrm{~N} 15 \mathrm{~K}_{2} \mathrm{O}\right.$ y $\left.15 \mathrm{P}_{2} \mathrm{O}_{5}\right)$.

Tabla 2. Composición (\%) de los fertilizantes químicos y compuestos

\begin{tabular}{|c|c|c|c|c|c|c|c|}
\hline Composición & $\begin{array}{l}\text { Boro } \\
\text { (FQ1) }\end{array}$ & $\begin{array}{l}\text { Urea } \\
\text { (FQ2) }\end{array}$ & $\begin{array}{l}\text { DAP* }^{*} \\
\text { (FQ3) }\end{array}$ & Composición & $\begin{array}{c}\text { Carbono } \\
\text { (FQ4) }\end{array}$ & $\begin{array}{c}\mathrm{KCl} \\
\text { (FQ5) }\end{array}$ & $\begin{array}{c}\text { Triple } \\
15^{\star} \\
\text { (FQ6) }\end{array}$ \\
\hline $\begin{array}{l}\text { Nitrógeno Total } \\
\text { (N) }\end{array}$ & 3 & - & & $\begin{array}{l}\text { Potasio total } \\
\left(\mathrm{K}_{2} \mathrm{O}\right)\end{array}$ & 1.7 & - & - \\
\hline $\begin{array}{l}\text { Nitrógeno ureico } \\
\text { (N) }\end{array}$ & 3 & - & & $\begin{array}{l}\text { Calcio total } \\
(\mathrm{CaO})\end{array}$ & 8 & - & - \\
\hline Azufre Total (S) & 6 & - & & $\begin{array}{l}\text { Carbono } \\
\text { orgánico } \\
\text { oxidable total }\end{array}$ & 14 & - & - \\
\hline Boro (B) & 2.5 & - & & Relación C/N & 14 & - & - \\
\hline Cobre $(\mathrm{Cu})$ & 0.5 & - & & $\begin{array}{l}\text { Humedad } \\
\text { máxima }\end{array}$ & 20 & - & - \\
\hline Silicio $\left(\mathrm{SiO}_{2}\right)$ & 17 & - & & $\mathrm{pH}$ en $10 \%$ & 8 & - & - \\
\hline Zinc $(Z n)$ & 15 & - & & Densidad a $20^{\circ} \mathrm{C}$ & 0.7 & - & - \\
\hline Nitrógeno & - & 46.0 & & $\begin{array}{l}\text { Cloruro de } \\
\text { potasio (KCl) }\end{array}$ & - & 96 & - \\
\hline Humedad & - & 0.5 & & Potasio $\left(\mathrm{K}_{2} \mathrm{O}\right)$ & - & 62 & - \\
\hline Biuret & - & 1.0 & & Agua $\left(\mathrm{H}_{2} \mathrm{O}\right)$ & - & 2 & - \\
\hline $\begin{array}{c}\text { Total de } \\
\text { nutrientes } \\
\left(\mathrm{N}+\mathrm{P}_{2} \mathrm{O}_{5}\right)\end{array}$ & - & - & 64 & $\begin{array}{c}\text { Contenido } \\
\mathrm{Ca}+\mathrm{Mg}\end{array}$ & - & 0.2 & - \\
\hline $\begin{array}{c}\text { Fósforo } \\
\text { pentóxido }\left(\mathrm{P}_{2} \mathrm{O}_{5}\right)\end{array}$ & - & - & 46 & $\begin{array}{l}\text { Sodio cloruro } \\
\text { (NaCl) }\end{array}$ & - & 1.2 & - \\
\hline Nitrógeno total & - & - & 18 & $\begin{array}{l}\text { Insoluble en } \\
\text { agua }\end{array}$ & - & 0.1 & - \\
\hline $\begin{array}{l}\text { Soluble en agua } \\
\qquad\left(\mathrm{P}_{2} \mathrm{O}_{5}\right)\end{array}$ & - & - & 41 & Sulfato $\mathrm{SO}_{4}$ & - & 0.5 & - \\
\hline \multirow[t]{5}{*}{ Humedad } & - & - & 2.5 & $\begin{array}{l}\text { Nitrógeno total } \\
(\mathrm{N})\end{array}$ & - & - & 15 \\
\hline & - & - & & $\begin{array}{c}\text { Nitrógeno } \\
\text { amoniacal (N) }\end{array}$ & - & - & 8.5 \\
\hline & - & - & & $\begin{array}{l}\text { Nitrógeno nítrico } \\
(\mathrm{N})\end{array}$ & - & - & 6.5 \\
\hline & - & - & & $\begin{array}{c}\text { Fosforo } \\
\text { asimilable } \\
\text { (P2O5) }\end{array}$ & - & - & 15 \\
\hline & - & - & & $\begin{array}{l}\text { Potasio soluble } \\
\text { en agua }\left(\mathrm{K}_{2} \mathrm{O}\right)\end{array}$ & - & - & 15 \\
\hline
\end{tabular}


Tabla 3. Aplicación de fertilizantes $(\mathrm{Kg} / \mathrm{ha})$ a las variedades de arroz

\begin{tabular}{|c|c|c|c|c|c|c|c|c|c|c|c|}
\hline & \multirow{3}{*}{$\mathbf{T}^{\star}$} & \multicolumn{4}{|c|}{ Variedad Victoria } & \multicolumn{6}{|c|}{ Variedad Fedearroz 68} \\
\hline & & \multicolumn{10}{|c|}{ Aplicaciones del fertilizante* } \\
\hline & & Primera & Segunda & Tercera & $\begin{array}{l}\text { Sub- } \\
\text { total }\end{array}$ & Primera & Segunda & Tercera & Cuarta & $\begin{array}{l}\text { sub } \\
\text { total }\end{array}$ & Total \\
\hline \multirow{4}{*}{1} & FO1 & 300 & 0 & 0 & 300 & 240 & 0 & 0 & 0 & 240 & 540 \\
\hline & FO2 & 0 & 400 & 800 & 1200 & 0 & 320 & 640 & 240 & 1200 & 2400 \\
\hline & FQ1 & 50 & 0 & 0 & 50 & 40 & 0 & 0 & 0 & 40 & 90 \\
\hline & FQ4 & 100 & 0 & 0 & 100 & 80 & 0 & 0 & 0 & 80 & 180 \\
\hline \multirow{4}{*}{2} & FO1 & 400 & 0 & 0 & 400 & 320 & 0 & 0 & 0 & 320 & 720 \\
\hline & FO2 & 0 & 400 & 600 & 1000 & 0 & 320 & 320 & 480 & 1120 & 2120 \\
\hline & FQ1 & 50 & 0 & 0 & 50 & 40 & 0 & 0 & 0 & 40 & 90 \\
\hline & FQ2 & 0 & 100 & 0 & 100 & 0 & 80 & 0 & 0 & 80 & 180 \\
\hline \multirow{4}{*}{3} & FQ1 & 50 & 0 & 0 & 50 & 40 & 0 & 0 & 0 & 40 & 90 \\
\hline & FQ2 & 100 & 100 & 100 & 300 & 80 & 80 & 80 & 80 & 240 & 540 \\
\hline & FQ3 & 100 & 0 & 0 & 100 & 80 & 0 & 0 & 0 & 80 & 180 \\
\hline & FQ5 & 100 & 100 & 100 & 300 & 80 & 80 & 80 & 80 & 240 & 540 \\
\hline \multirow{2}{*}{4} & FQ1 & 50 & 0 & 0 & 50 & 40 & 0 & 0 & 0 & 40 & 90 \\
\hline & FQ6 & 400 & 400 & 600 & 1400 & 320 & 320 & 480 & 240 & 1360 & 2760 \\
\hline \multirow{4}{*}{5} & FO1 & 200 & 0 & 0 & 200 & 160 & 0 & 0 & 0 & 160 & 360 \\
\hline & FQ1 & 50 & 0 & 0 & 50 & 40 & 0 & 0 & 0 & 40 & 90 \\
\hline & FQ2 & 100 & 100 & 200 & 400 & 80 & 80 & 160 & 150 & 470 & 870 \\
\hline & FQ5 & 0 & 100 & 200 & 300 & 0 & 80 & 160 & 150 & 390 & 690 \\
\hline \multirow{6}{*}{6} & FO1 & 0 & 200 & 0 & 200 & 0 & 160 & 0 & 0 & 160 & 360 \\
\hline & FO2 & 0 & 0 & 400 & 400 & 0 & 0 & 320 & 200 & 520 & 920 \\
\hline & FQ1 & 50 & 0 & 0 & 50 & 40 & 0 & 0 & 0 & 40 & 90 \\
\hline & FQ2 & 0 & 50 & 100 & 150 & 0 & 40 & 80 & 80 & 200 & 350 \\
\hline & FQ3 & 300 & 0 & 0 & 300 & 240 & 0 & 0 & 0 & 240 & 540 \\
\hline & FQ5 & 100 & 0 & 100 & 200 & 80 & 0 & 80 & 80 & 240 & 440 \\
\hline
\end{tabular}

*FO1= fertilizante orgánico 1 (N orgánico); FO2= fertilizante orgánico 2 (N ureico); FQ1= fertilizante químico 1 (boro); FQ2=fertilizante químico 2 (urea); FQ3= fertilizante químico $3\left(\mathrm{DAP}^{\star \star}\right)$; FQ4= fertilizante químico 4 (carbono); FQ5= fertilizante químico $5\left(\mathrm{KCl}\right.$ ) y FQ6= fertilizante químico 6 (triple $15^{\star \star}$ ). $\mathrm{T}$ = tratamiento: $1=45 \%$ orgánico con carbono; $2=90 \%$ orgánico sin carbono; $3=100 \%$ químico con urea (tradicional); $4=100 \%$ químico sin urea (otra alternativa tradicional); $5=45 \%$ orgánico con DAP y $6=90 \%$ orgánico sin DAP.

${ }^{*}$ DAP $=$ P2O 5 y triple $15=15 \mathrm{~N}, 15 \mathrm{~K} 2 \mathrm{O}$ y $15 \mathrm{P} 2 \mathrm{O} 5$. 


\section{Diseño experimental}

Como el trabajo se realizó bajo condiciones controladas, se utilizó un diseño completamente al azar, con cinco repeticiones por cada tratamiento, evaluando seis tratamientos; los datos obtenidos en las diferentes evaluaciones se trabajaron por medio de un análisis de varianza y posterior comparación de medias por test de Duncan, teniendo en cuenta los tratamientos, la zona y las diferentes épocas en que se realizaron las evaluaciones.

Para la recolección de las muestras se construyeron marcos de $2 \times 3\left(6 \mathrm{~m}^{2}\right)$ para ubicar en todos los bloques del ensayo las dos variedades; se cortaron las panículas presentes en el interior de los marcos ubicados en los bloques, para así determinar peso de grano, además se recogieron 10 panículas por bloque para evaluar peso de grano lleno y vaneado, y así estimar porcentaje de vaneamiento y manchado en grano; también se estimó el porcentaje de incidencia de los siguientes patógenos: Sarocladium oryzae, Helminthosporium, ácaros, Gaenomices, Pyricularia oryzae y virus hoja blanca (VHB).

\section{RESULTADOS Y DISCUSIÓN}

Al comparar el rendimiento de los seis tratamientos en la variedad Fedearroz 68 (VF68) se observa, que el tratamiento con rendimiento más alto $(\mathrm{P}<0.05)$ fue el de $45 \%$ orgánico sin $\mathrm{P}_{2} \mathrm{O}_{5}$ con $6390 \mathrm{~kg} / \mathrm{ha}$, seguido por el tratamiento con $90 \%$ orgánico con urea (5780 kg/ha), siendo el de la producción más baja, el que se le aplicó $90 \%$ orgánico con $\mathrm{P}_{2} \mathrm{O}_{5}$; esto implica que la adición de urea en la mezcla fue más provechosa y asimilada por la planta que la de DAP, la cual contiene $\mathrm{P}_{2} \mathrm{O}_{5}$, (Tablas 2 y 3, Gráfica 1). Por otro lado, al promediar las evaluaciones que se realizaron en la variedad victoria 10-38 (VV 10-39) para determinar el rendimiento resultante, se observó que el tratamiento $100 \%$ químico sin cloruro de potasio $(\mathrm{KCl})$ produjo $8200 \mathrm{~kg} / \mathrm{ha}$, siendo este el de mayor rendimiento $(\mathrm{P}<0.05)$, demostrándose que no se requiere la adición de $\mathrm{KCl}$, urea, ni $\mathrm{P}_{2} \mathrm{O}_{5}$ (DAP); el tratamiento que le siguió fue el que contuvo 90\% orgánico y $\mathrm{P}_{2} \mathrm{O}_{5}(7800 \mathrm{~kg} / \mathrm{ha})$, 
este último componente si afectó positivamente la producción en VV10-39, más no en VF 68 Kg/ha (Tabla 3 y Gráfica 1).

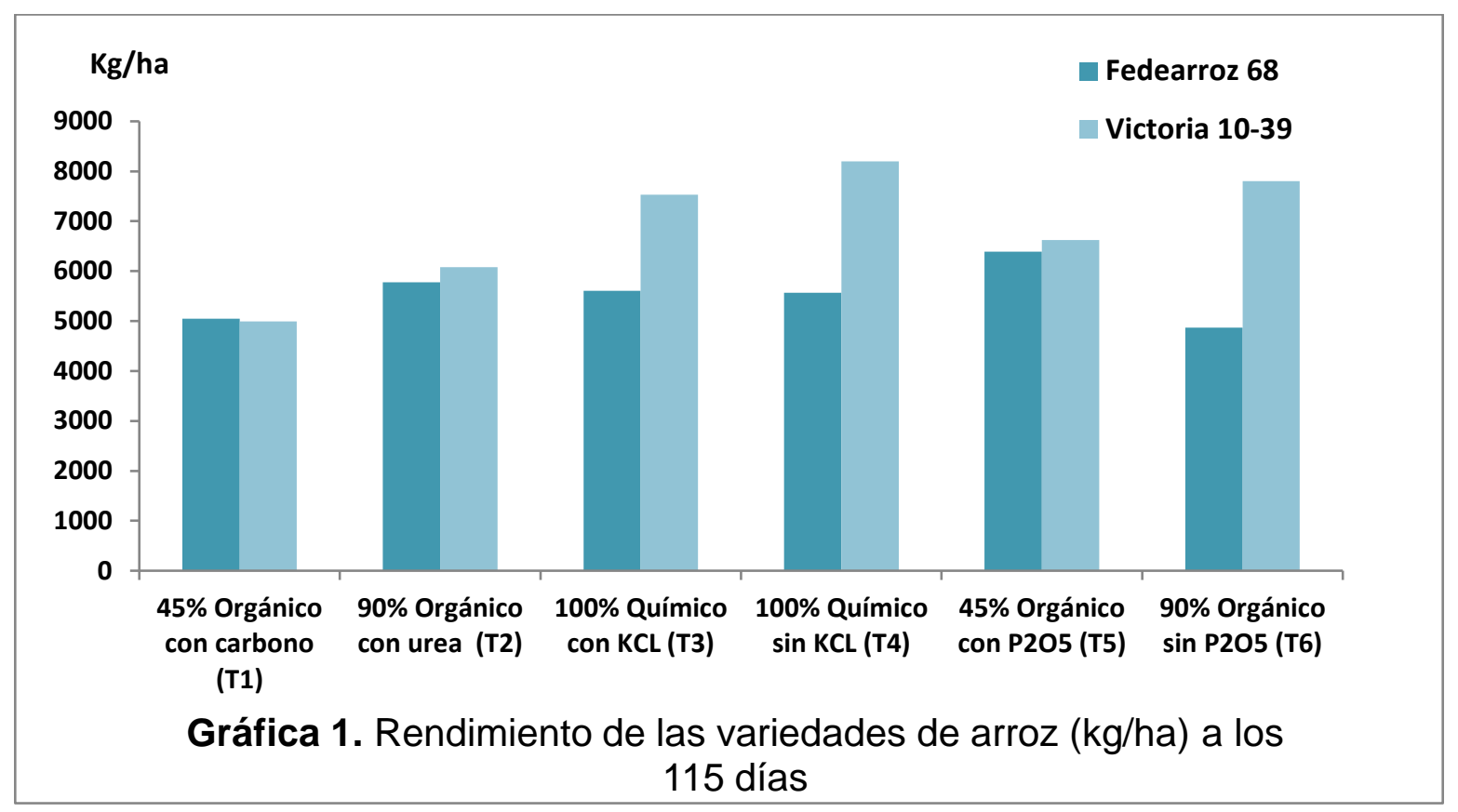

En las dos variedades de arroz, las plantas del T1 fueron las de menor vigor híbrido $(\mathrm{P}<0.05)$ con respecto a los otros tratamientos, por otro lado, T2 y T6 mostraron los mayores porcentajes de valor hibrido, sin que se presentaran diferencias estadísticas con los demás tratamientos $(P>0.05)$, lo cual significa que las mezclas de fertilizantes en los cinco tratamientos, tienen un efecto similar para esta variable (Tabla 3 y Gráfica 2).

Como se puede observar en la VF68 se presentaron las mayores longitudes de las plantas en los tratamientos 2, 4 y 6 , siendo la más baja $T 5(50 \mathrm{~cm})(P<0.05)$, lo cual significa que la adición $\mathrm{P}_{2} \mathrm{O}_{5}$ es importante para el crecimiento de las plantas de esta variedad. Respecto a la evaluación de altura en el transcurso del desarrollo del cultivo de VV 10-39 los tratamientos 3,4 y 5 presentaron una similitud en sus alturas, pero en los tratamientos 1 y 2 su crecimiento fue menor $(\mathrm{P}<0.05)$, lo cual está relacionado con el vigor híbrido (Gráfica 2) porque se observan comportamientos similares en estas dos variables de respuestas analizadas (Tabla 3 y Gráfico 3). 

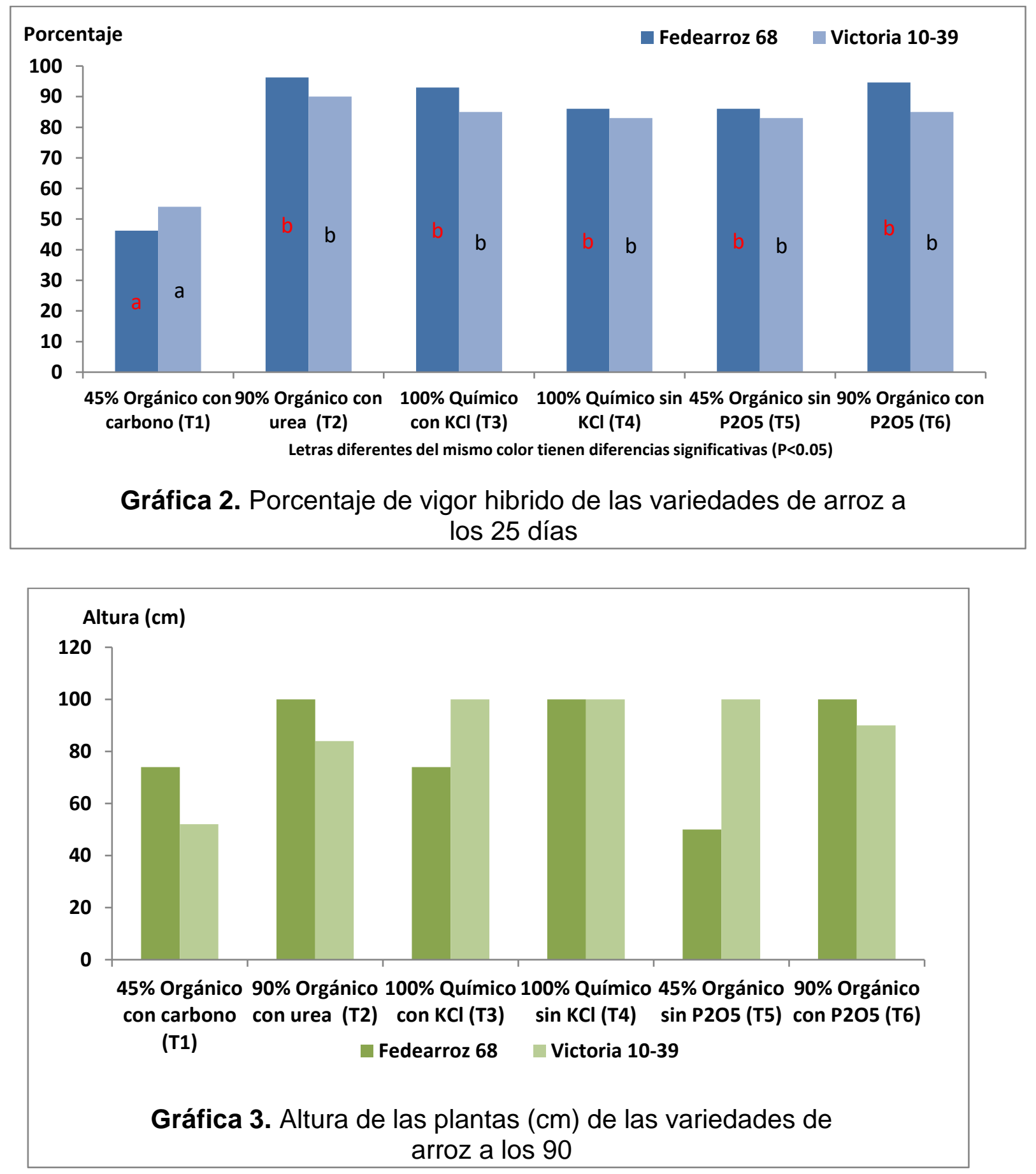

En la VF 68 se puede observar que hay menor porcentaje de granos manchados los tratamientos 1,2 y 5 , los cuales fueron bajos $(P<0.05)$ con respecto a los demás tratamientos, es de anotar que los tratamientos con los menores granos manchados fueron fertilizados con urea y boro, lo más factible es que esta mezcla favorezca la presentación del producto (Tabla 3 y Gráfica 4). En contraste con la 
Gráfica 1 (rendimiento) los tratamientos 5 y 2 muestran ser más resistentes al manchado del grano; mientras que en la VV10-38 el porcentaje de grano manchado fue menor ( $P>0.05)$ en T2 con el $6.2 \%$, seguido de $\mathrm{T} 1$ y $\mathrm{T} 4$, después T5 y por último T6 con el $27 \%$ de grano manchado, en T2 se observó el efecto positivo que tiene el abono orgánico mezclado con boro y urea para mantener las calidades organolépticas del grano de arroz en esta variedad (Tabla 3 y Gráfica 4).

En la evaluación de la decoloración del grano en VF 68, se puede observar que el porcentaje de granos afectados fue menor para los tratamientos 1,3 y 5 en comparación a 2, 4 y 6, mostrando una diferencia significativa $(P<0.05)$. Se puede observar que sigue el mismo patrón respecto al rendimiento en la Gráfica 1, donde T5 muestra mejor color, exceptuando T2 que tuvo buen rendimiento pero alto porcentaje de decoloración. En VV 10-38 la decoloración del grano, fue superior en T6 (9.2\%), con una gran diferencia en comparación a los otros tratamientos, mientras que T3 presentó el menor porcentaje en decoloración del grano (2.8\%) $(\mathrm{P}<0.05)$. Respecto a T4 que representa el mayor rendimiento (Gráfica 1 ) es el que sigue en menos promedio de manchado de grano junto con los tratamientos 1 y 2 (Gráfica 4).

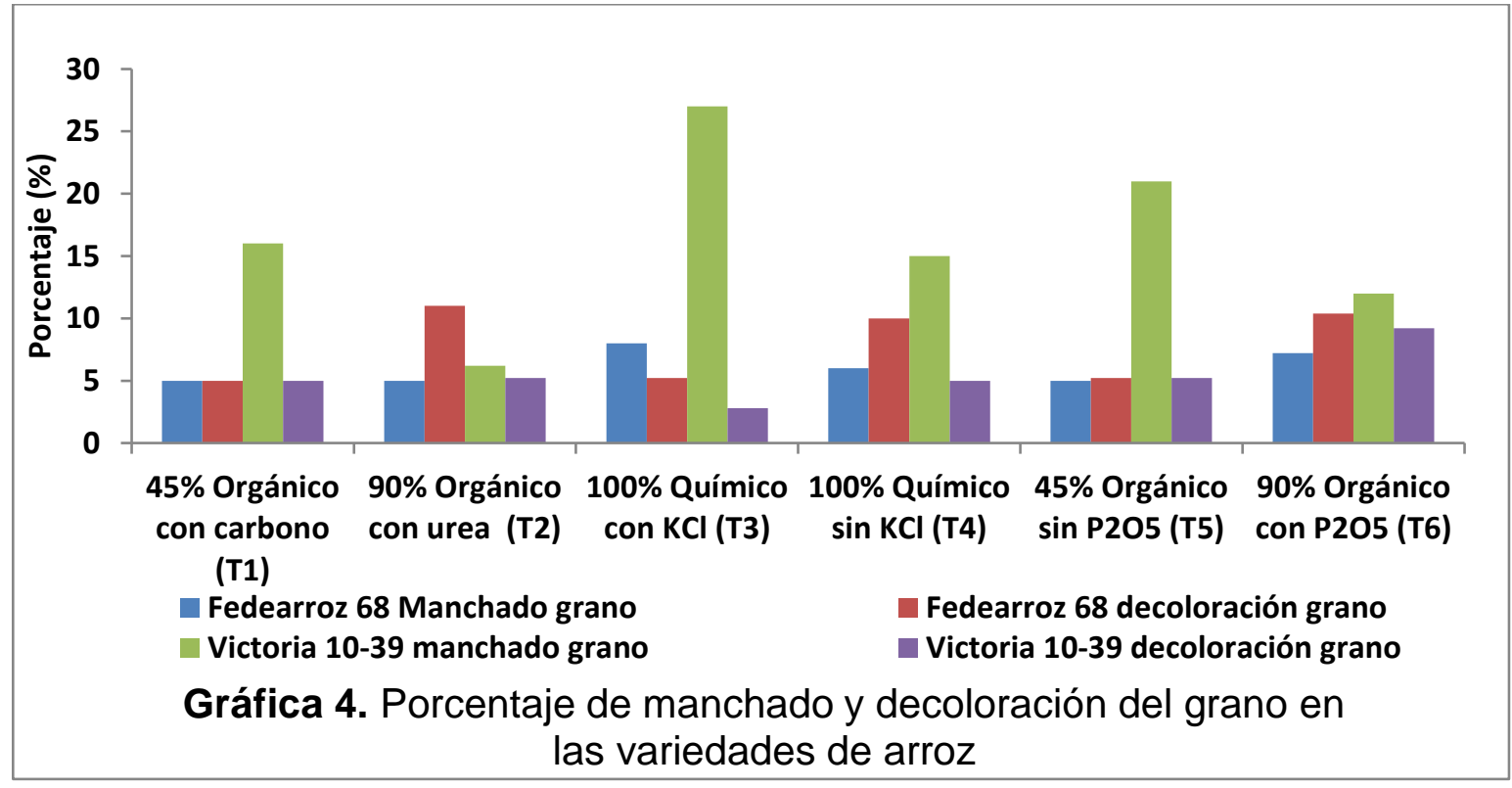


En la VF 68 T4 y T6 presentaron mayor porcentaje de vaneamiento $(P<0.05)$ en comparación con los otros tratamientos, es importante resaltar que, aunque T4 no fue de los mejores en la producción, sí presentó el peso más alto en granos lleno, mostrando una alta diferencia en comparación de los otros tratamientos $(P<0.05)$. Se puede observar que T1 y T2, que tienen los fertilizantes orgánicos, son los que tienen mayor porcentaje de granos llenos más del 97\% (vaneamiento menos del $3 \%)$. En VV 10-39, T1 fue el más alto $(P<0.05)$ en porcentaje vaneamiento $(12 \%)$, seguido de T6 (10\%) y T2 (8\%). Se observa que la adición del boro, que está presente en T3, T4 y T5, puede estar influyendo en la disminución de los granos vanos ( $4 \%$ o menos), por lo tanto, este componente es importante para obtener un buen porcentaje de granos llenos en VV 10-39 (Tabla 3 y Gráfica 5).

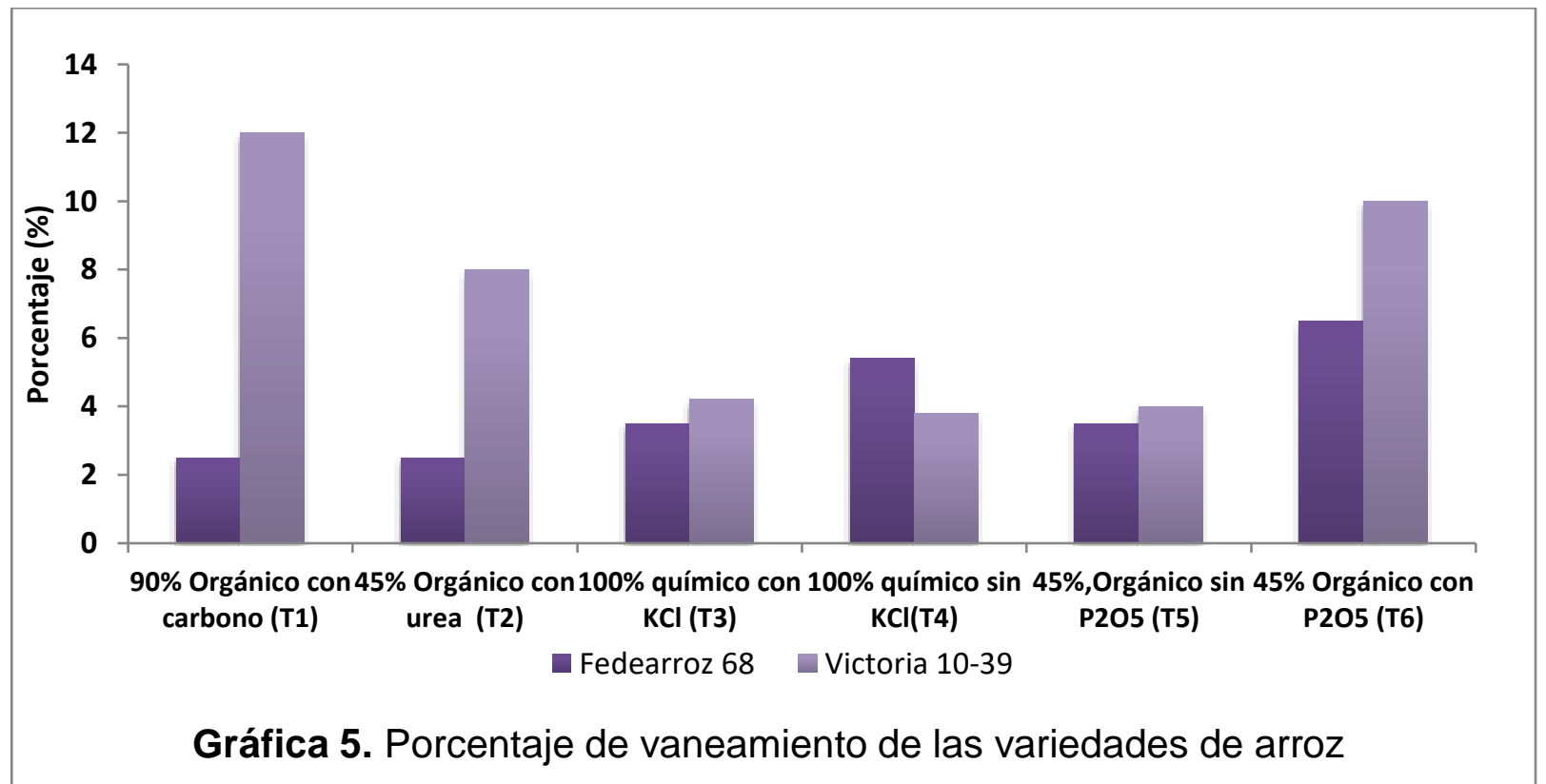

Sarocladium oryzae. afectó en mayor proporción VV 10-39 en comparación a VF 68, principalmente en T2 (21.3\%), T3 (15.4\%) y T6 (12.6\%), mientras que el mayor ataque de esta enfermedad en VF 10-39 no paso del $8.4 \%$ en T6, con lo cual se deduce que esta última variedad de arroz, es más resistente que la primera, a esta enfermedad principalmente en T5, que contiene el $45 \%$ de orgánico y DAP $\left(\mathrm{P}_{2} \mathrm{O}_{5}\right)$, (Tablas 3 y 4). Mediante sucesivas evaluaciones de campo desde 1991, se ha observado un incremento considerable en la incidencia Sarocladium oryzae, la VV 
10-39 fue la que más daño sufrió en T2, T5 y T6 por este virus, que ocasiona pudrición de la vaina del arroz. Cuando las infecciones tienen lugar en la hoja bandera durante la etapa de emergencia de la espiga (embuchamiento), provocan anomalías en la panícula en formación; en algunos casos, se puede observar que las panículas no emergen o lo hacen parcialmente, mientras las glumas tienden a dañarse y toman una coloración marrón oscuro o rojizo, provocando granos vanos o parcialmente llenos y arrugados, afectando la calidad y cantidad de la cosecha (Cardona, 2013).

De Helminthosporium se puede resaltar que en VF 68 las plantas sometidas a los tratamientos 5 y 2 fueron menos susceptibles a este hongo (4.6 y $5.0 \%$ ) en comparación a los demás tratamientos que mostraron diferencias significativas en especial T6 que fue el más afectado $(P<0.05)$, mientras que en la VV10-39 fue T1 el más susceptible (20\%) y los menos fueron T4 (1.6\%) y T6 $(2.0 \%)(\mathrm{P}<0.05)$, esto implica que la variedad de arroz tiene una mayor resistencia genética a esta enfermedad, siendo este factor más influyente que el tratamiento (Tabla 4). Se observó que este patógeno afecto con más del 10\% cuatro tratamientos, en VF 68 el 1 y 6 y en VV 10-39 el 1 y 2 , lo cual es considerado como una incidencia baja, puesto que el complejo Helminthosporium en el que se incluyen varias especies de hongos es bastante agresivo deteriorando raíz, tallo, hojas, panículas y granos de arroz, también en las hojas del arroz se observan lesiones pequeñas, circulares y de color marrón, mientras que en las vainas se presentan pequeños puntos ovales acuosos de color verde oliva con un halo amarillo (Cardona y González, 2008).

Ácaros, en la mayoría de los tratamientos su incidencia fue baja en las dos variedades de arroz, sin embargo, VF68 T1 presenta una diferencia significativa con los demás tratamientos $(P<0.05)$, siendo éste el mayor porcentaje de ácaros encontrados, lo que justifica su menor rendimiento (Gráfica 1). Es de anotar que T4 presentó el porcentaje más alto de rendimiento en VV 10-39, mostrando el porcentaje más bajo de incidencia de ácaros; aunque no se observaron diferencias con T3, T5 y T6 (P>0.05) (Tabla 4). La detección temprana del ácaro disminuye el 
riesgo de incremento de las poblaciones en el campo, por lo tanto, se debe utilizar semilla certificada evitando prácticas que causen estrés al cultivo, tales como: suelos compactados, aplicaciones tardías de herbicidas, desbalance en la fertilización, mal manejo del agua. Además se debe proteger y promover el incremento de poblaciones de los agentes del control biológico ya que estos ácaros ocasionan perdidas (Rippstein et al., 2001). Se puede afirmar que las parcelas experimentales estuvieron manejadas adecuadamente puesto que la incidencia de esta enfermedad no fue mayor a $15 \%$ solamente en T1, también fue este tratamiento el de mayor porcentaje de vaneamiento (Gráfica 5) en comparación con los demás, esta incidencia no fue mayor del 11.1\% (Tabla 4).

Tabla 4. Incidencia de enfermedades (\%) en las variedades de arroz

\begin{tabular}{|c|c|c|c|c|c|c|c|c|c|c|c|c|}
\hline \multirow{2}{*}{$\mathrm{T}$} & \multicolumn{2}{|c|}{$\begin{array}{l}\text { Sarocladium } \\
\text { oryzae }\end{array}$} & \multicolumn{2}{|c|}{ Helminthosporium } & \multicolumn{2}{|c|}{ Ácaros } & \multicolumn{2}{|c|}{ Gaenomices } & \multicolumn{2}{|c|}{$\begin{array}{c}\text { Pyricularia } \\
\text { oryzae }\end{array}$} & \multicolumn{2}{|c|}{$\begin{array}{l}\text { Virus hoja } \\
\text { blanca }\end{array}$} \\
\hline & $\begin{array}{l}\text { VF } \\
68\end{array}$ & $\begin{array}{c}\text { V V } \\
10-39\end{array}$ & VF 68 & $\begin{array}{c}\text { V V } \\
10-39\end{array}$ & $\begin{array}{l}\text { VF } \\
68\end{array}$ & $\begin{array}{c}\text { V V } \\
10-39\end{array}$ & $\begin{array}{l}\text { VF } \\
68\end{array}$ & $\begin{array}{c}\text { V V } \\
10-39\end{array}$ & $\begin{array}{l}\text { VF } \\
68\end{array}$ & $\begin{array}{c}\mathrm{V} V \\
10-39\end{array}$ & $\begin{array}{l}\text { VF } \\
68\end{array}$ & $\begin{array}{c}\mathrm{VV} \\
10-39\end{array}$ \\
\hline 1 & $5.2^{b}$ & $3.1^{d}$ & $11^{c}$ & $20^{d}$ & $10^{\mathrm{b}}$ & $15^{c}$ & $13.4^{c}$ & $12.2^{\mathrm{c}}$ & $11.8^{\mathrm{c}}$ & $10.6^{c}$ & $5^{\mathrm{ab}}$ & $0^{a}$ \\
\hline 2 & $3.8^{\mathrm{ab}}$ & $21.6^{c}$ & $5.0^{\mathrm{a}}$ & $16^{c}$ & $5^{a}$ & $10.1^{\mathrm{b}}$ & $5^{a}$ & $7.5^{\mathrm{b}}$ & $4.6^{\mathrm{ab}}$ & $5.5^{b}$ & $0^{\mathrm{a}}$ & $0^{\mathrm{a}}$ \\
\hline 3 & $5.0^{\mathrm{ab}}$ & $15.4^{b}$ & $8.0^{\mathrm{b}}$ & $7.6^{\mathrm{b}}$ & $6^{\mathrm{ab}}$ & $5^{a}$ & $8.4^{\mathrm{b}}$ & $5^{a}$ & $4.6^{\mathrm{ab}}$ & $5^{b}$ & $0^{\mathrm{a}}$ & $0^{\mathrm{a}}$ \\
\hline 4 & $5.0^{\mathrm{ab}}$ & $9.6^{\mathrm{a}}$ & $8.4^{\mathrm{b}}$ & $1.6^{\mathrm{a}}$ & $8.3^{\mathrm{ab}}$ & $4.5^{\mathrm{a}}$ & $10^{b}$ & $5^{a}$ & $5^{\mathrm{ab}}$ & $2^{a}$ & $2^{\mathrm{ab}}$ & $2^{\mathrm{a}}$ \\
\hline 5 & $2.0^{\mathrm{a}}$ & $20^{c}$ & $4.6^{\mathrm{a}}$ & $7.8^{\mathrm{b}}$ & $5.6^{a}$ & $5^{a}$ & $4.6^{\mathrm{a}}$ & $9.2^{c}$ & $2^{a}$ & $5.5^{b}$ & $0^{\mathrm{a}}$ & $5^{\mathrm{a}}$ \\
\hline 6 & $8.4^{c}$ & $12.6^{\mathrm{ab}}$ & $15^{d}$ & $2.0^{\mathrm{a}}$ & $9^{a b}$ & $5^{a}$ & $25^{\mathrm{d}}$ & $5^{\mathrm{a}}$ & $7.2^{b}$ & $5^{b}$ & $6^{b}$ & $2^{\mathrm{a}}$ \\
\hline
\end{tabular}

Letras diferentes en la misma fila indican que hay significancia de $\mathrm{P}<0.05$

${ }^{*} \mathrm{~T}=$ tratamiento: $1=45 \%$ de orgánico con carbono; $2=90 \%$ de orgánico sin carbono; $3=100 \%$ químico con urea (tradicional); $4=100 \%$ químico sin urea (otra alternativa tradicional); $5=45 \%$ orgánico con DAP y $6=90 \%$ orgánico sin DAP. ${ }^{*} \mathrm{DAP}=\mathrm{P}_{2} \mathrm{O}_{5}$ y triple $15=15$ de $\mathrm{N} .15 \mathrm{~K}_{2} \mathrm{O}$ y $15 \mathrm{P}_{2} \mathrm{O}_{5}$.

VF 68 $=$ Variedad Fedearroz 68. VV 10-39= Variedad Victoria 10-39.

Respecto a Gaenomices en VF68 se logra observar como las plantas fertilizadas con los tratamientos 5 y 2 presentaron mayor resistencia a este patógeno, por otro lado tratamientos como el 1 y 6 siguen demostrando que no son muy eficientes en el momento de darle a la planta los nutrientes necesarios para que esta adquiera mayor resistencia a patógenos; los tratamientos 1 y 2 muestran no diferencias significativas con los otros $(P>0.05)$, en especial con $T 6$, que demostró tener un porcentaje de ataque alto (Tabla 4). En VV 10-39 se encontró que T1 sigue siendo el de mayor susceptibilidad a Gaenomices con una diferencia significativa con los otros tratamientos $(\mathrm{P}<0.05)$, con lo cual se puede explicar sus bajas producciones, 
(Gráfica 1), a su vez que T3, T4 y T6 fueron los de menor incidencia, de esta manera se corrobora con el buen rendimiento de T4 y T6, también se observó que la incidencia a este hongo fue baja en la mayoría de tratamientos de las dos variedades, exceptuando T6 en VF68 (25\%) (Tabla 4). Se realizaron estrategias de prevención y control para este patógeno según recomendaciones de Ospina, (2009) aplicando practicas integrales, contemplando un buen manejo agronómico del cultivo, en lo cual se incluyó preparación del terreno dejando descomponer residuos de cosechas anteriores, y se aplicó un buen programa de fertilización, que fueron los tratamientos descritos evitando estresar el cultivo, buscando mejorar la microbiología del suelo con el uso de abonos orgánicos en algunos tratamientos.

En cuanto a Pyricularia oryzae se pudo evidenciar en VF 68 que las plantas sometidas a los tratamientos 2, 3, 4 y 5 fueron las menos susceptibles al ataque de este hongo, al contrario, en $\mathrm{T} 1$ se observó una mayor incidencia $(\mathrm{P}<0.05)$. En esta evaluación se presentó algo notable, T6 no fue el peor y T2 que es resistente a otras enfermedades no mostró diferencias significativas con otros tratamientos ( $P>0.05)$ (Tabla 4). En VV 10-39 la presencia de Pyricularia oryzae fue mayor en $\mathrm{T} 1$, a su vez T4 tiene el menor porcentaje de incidencia de este hongo $(\mathrm{P}<0.05)$, lo cual coincide con su alto rendimiento (Gráfica 1 y Tabla 4). Esta enfermedad se considera un factor biótico adverso al cultivo, puesto que quema el arroz, causando pérdidas económicas hasta del $100 \%$, se puede afirmar que las variedades de arroz de este experimento son resistentes a este hongo, porque su ataque no fue mayor al $12 \%$.

Con respecto al virus de la hoja blanca (Tagosodes orizicolus) (VHB), en VF 68 se pudo observar que solo las plantas de T1, T4 y T6 presentaron ataque por el este virus $(P<0.05)$, mientras que en VV10-39, los tratamientos afectados fueron el 4, 5 , y 6 con diferencias significativas $(P<0.05)$ en comparación con los tratamientos 2 , 3 y 5 , que no mostraron ser atacados por este virus. En general estas dos variedades mostraron altos porcentajes de resistencia al ataque por el virus de la hoja blanca, puesto que no fue mayor al 5\% (Tabla 4), siendo importante resaltar 
que las variedades difieren en su nivel de resistencia al VHB por factores genéticos, lo cual es un instrumento valioso para la reducción de los efectos negativos de esta enfermedad; muchas variedades comerciales de arroz son resistentes al daño mecánico causado por las sogatas al alimentarse de la planta, pero no tienen un adecuado nivel de resistencia al VHB. Las plantas resistentes que empiezan a ser infectadas en edades muy tempranas con VHB son severamente afectadas; y para poder controlar el VHB se requieren, tanto variedades resistentes, como buenas prácticas de manejo (Labrin, 2007).

\section{CONCLUSIONES}

Los componentes de rendimiento que se analizaron suministraron datos que permitieron concluir que la fertilización realizada en T4 en VV 10-39 es la que mejor aporte nutricional dio a la planta, generando un óptimo desarrollo del cultivo permitiéndole ser el tratamiento con mayor tolerancia a los problemas fitosanitarios que se presentaron, mientras que en T1 la fertilización no fue suficiente para generar una buena nutrición y así afrontar los problemas fitosanitarios a la vez que su desarrollo fisiológico fue retrasado y débil. En VF 68 el T5 mostro los mejores resultados en todas las variables evaluadas, en tanto que T6 fue el de menor rendimiento y fue susceptible a la mayoría de problemas fitosanitarios.

La enfermedad que mayor incidencia tuvo fue la provocada por el hongo Sarocladium oryzae, en todos los tratamientos realizados en VV 10-39 y la que menor incidencia tuvo fue la del virus de hoja blanca (VHB), además se observó que independiente de los tratamientos hubo tolerancia similar de Pyricularia oryzae a excepción de T1, que mostro la mayor incidencia con un 10\%. En VF 68 Gaenomices fue la enfermedad que más incidió en todos los tratamientos evaluados, y la que mostro menos incidencia fue el virus de la hoja blanca.

En VV10-39 el mejor rendimiento se observó en T4, siendo el que mejor permitió expresar las características de la variedad para afrontar los problemas fitosanitarios ofreciendo mayor cantidad de granos de arroz. En la VF 68, el mayor 
rendimiento fue visto en T5 proporcionando a las plantas los nutrientes necesarios para su buen desarrollo y tolerancia a problemas fitosanitarios.

\section{REFERENCIAS BIBLIOGRÁFICAS}

1. Cardona R. Sarocladium oryzae: Agente causal de la pudrición de la vaina del arroz en Venezuela. Revista de la Sociedad Venezolana de Microbiología. 33 (1): 80-82. 2013.

2. Cardona R., González M.S. Caracterización y patogenicidad de hongos del complejo Helminthosporium asociados al cultivo del arroz en Venezuela. Bioagro. 20 (2): 141-145. 2008.

3. Castilla L.A., Tirado Y.C., Cuellar J.C. Manejo agronómico por ambiente, el primer paso en la agricultura de precisión. Revista Arroz. 65 (527): 14-20. 2017.

4. Chica J., Tirado Y.C., Barreto J.M. Indicadores de competitividad del cultivo del arroz en Colombia y Estados Unidos. Revista de Ciencias Agrícolas. 33 (2): 16-31. 2016.

5. Duwayri M., Van Tran D. Reflections on yield gaps in rice production: how to narrow the gaps. RAP Publication (FAO), Boletín de la Comisión Internacional del Arroz. 49 12-25. 1999.

6. FEDEARROZ, Federación Nacional de Arroceros. Dinámica del sector arrocero de los Llanos Orientales de Colombia 1999-2011. Bogotá DC, Colombia, 1a Ed. 159 p. 2011.

7. FEDEARROZ, Federación Colombiana de Arroceros. Adopción masiva de Tecnología. Bogotá DC, Colombia, 29 p. 2015.

8. Labrin N.Y. Estudio de la resistencia en variedades de arroz (Oryza sativa L.) venezolanas al virus de la hoja blanca, Magister en Agricultura Ecológica. Programa de Educación para el Desarrollo y la Conservación, Escuela de Posgraduados, 84 p. 2007.

9. Ospina J. Alternativas de Control de la Mancha Naranja (Gaeumannomyces graminis var. graminis). Revista Arroz. 57 (479): 129-134. 2009.

10. Rippstein G., Escobar G., Motta F.M. Agroecología y biodiversidad de las sabanas en los Llanos Orientales de Colombia. CIAT, Cali, Colombia. 302 p. 2001.

11. Tinoco R., Acuña A., Cultivo de arroz (Oryza sativa). Manual de recomendaciones técnicas. San José, Costa Rica, 75 p. 2009. 